Cahiers $d u$ MONDE RUSSE

\section{Cahiers du monde russe}

Russie - Empire russe - Union soviétique et États indépendants

\section{$56 / 1 \mid 2015$}

Fictions d'avenir : sciences et temps des socialismes est-européens

\title{
Rapatriés et expulsés
}

Le retour des réfugiés de guerre juifs en Ukraine soviétique, entre exigences étatiques et urgences humanitaires (1919-1924)

Repatriated and deported: the return of Jewish war refugees in Soviet Ukraine, between state demands and humanitarian emergencies (1919-1924)

\section{Thomas Chopard}

\section{(2) OpenEdition \\ Journals}

Édition électronique

URL : http://journals.openedition.org/monderusse/8174

DOI : 10.4000/monderusse. 8174

ISSN : $1777-5388$

Éditeur

Éditions de l'EHESS

Édition imprimée

Date de publication : 1 janvier 2015

Pagination : 175-198

ISBN : 978-2-7132-2475-1

ISSN : $1252-6576$

\section{Référence électronique}

Thomas Chopard, «Rapatriés et expulsés », Cahiers du monde russe [En ligne], 56/1 | 2015, mis en ligne le 13 juillet 2019, consulté le 09 octobre 2020. URL : http://journals.openedition.org/monderusse/8174 ; DOI : https://doi.org/10.4000/monderusse.8174

Ce document a été généré automatiquement le 9 octobre 2020.

(c) École des hautes études en sciences sociales 


\section{Rapatriés et expulsés}

Le retour des réfugiés de guerre juifs en Ukraine soviétique, entre exigences étatiques et urgences humanitaires (1919-1924) ${ }^{1}$

Repatriated and deported: the return of Jewish war refugees in Soviet Ukraine, between state demands and humanitarian emergencies (1919-1924)

\section{Thomas Chopard}

1 Dans un rapport adressé en 1921 à la Conférence sur les réfugiés russes tenue à Genève sous les auspices de la Société des Nations, Lucien Wolf, au nom de plusieurs organismes d'assistance juifs européens, tente d'attirer l'attention des instances internationales sur le sort des Juifs d'Europe orientale. Il cherche à faire reconnaître la singularité du sort des Juifs de l'ancien Empire russe dans le cadre des mouvements de populations qui affectent les débris des empires européens après la Première Guerre mondiale. Dans ces chassés-croisés où se mêlent prisonniers de guerre et réfugiés de la Grande Guerre et de la guerre civile, l'Ukraine est un lieu de convergence de l'ensemble des flux et une porte vers l'Europe. La Société des Nations et son organisme dédié, le Haut Commissariat aux réfugiés (HCR) dirigé par le Dr Fridthof Nansen, n'établissent pas, ou peu, de distinction entre tous ces « réfugiés russes »:

Durant les douze derniers mois, il y eut un exode panique des Juifs d'Ukraine vers la Pologne et les pays frontaliers. Ils sont les fugitifs d'une indescriptible anarchie et d'un carnage qui a déjà ensanglanté des centaines de centres de vie juive et, qui plus est, a décimé la population locale. [...] Il y a par conséquent en Pologne une concentration considérable de réfugiés juifs - avoisinant les cent mille âmes -

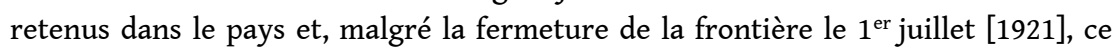
nombre a probablement augmenté.

Dans ces circonstances, le Gouvernement polonais s'est trouvé contraint de prendre des mesures, non seulement pour protéger le pays d'un nouvel afflux, mais aussi pour venir à bout de la présence des réfugiés qui sont à présent massés sur le territoire polonais, qui menacent de devenir un fardeau intolérable et épuisent déjà sévèrement les ressources de la République. ${ }^{2}$

2 L'empreinte de la guerre, l'urgence humanitaire et les frictions déjà vives avec l'État transparaissent dans tous les rapports. La Pologne et la Roumanie, les deux pays frontaliers de l'Ukraine soviétique, présentent toutefois une configuration particulière. 
Les populations massées dans ces pays ne sont pas dans la situation des exilés de Constantinople, partis par milliers en vagues successives et dont le départ sonne comme un adieu. Ayant fui les conséquences catastrophiques des guerres, les réfugiés aux frontières sont dans l'expectative. Les témoignages reflètent un parcours de plusieurs mois, empreint d'incertitude et d'improvisation. La frontière reste difficile à franchir. Le Dniestr notamment est un obstacle à l'entrée en Roumanie. Cependant, de part et d'autre de la frontière, la situation sanitaire est similaire : les réfugiés, souvent partis dans la précipitation, dépouillés de presque tous leurs biens, se nourrissent d'expédients, souvent grâce à l'aide humanitaire. La rupture n'est pas aussi nette qu'à Constantinople et la distinction entre réfugié temporaire et exilé définitif est souvent impossible à établir.

3 Toutefois, l'Ukraine et bientôt l'Union soviétique ne sont pas seulement des pays d'où se déversent des flots de réfugiés fuyant le bolchevisme et les ravages de la guerre civile. Avec l'arrêt des combats et l'extinction de la famine de 1921-1922, des réfugiés, épuisés par des conditions de vie tout juste supportables, prennent le parti de revenir. L'Ukraine met ainsi tôt en place, en harmonie avec la Russie, des politiques particulières de retour et d'accueil.

Cette vague de retours n'est pas le seul fait des Soviétiques. En Pologne et en Roumanie, gouvernements et autorités militaires font preuve, dès 1921, d'une intolérance grandissante à l'égard des réfugiés sur leur territoire, des juifs en particulier. Pour ceux-ci, la distinction est désastreuse entre «bons » réfugiés qualifiés de politiques, partisans des armées antibolcheviques ou partis dans leur sillage, et ces populations civiles fuyant la guerre et la persécution antisémite des pogroms qui ravagent alors l'Ukraine. La gestion des Juifs réfugiés hors d'Ukraine joue alors sur plusieurs plans. Elle met tout d'abord en lumière le statut des minorités juives au sein de ces États-nations fondés ou élargis après la révolution et, de fait, constitue un nouveau palier dans l'escalade de la violence imposée aux civils, déjà entamée en 1915. Elle introduit aussi une nouvelle phase, diplomatique et non plus armée, dans les relations avec le pouvoir soviétique. Face à l'afflux des réfugiés, les États frontaliers prennent en effet le parti de les chasser, soit par le biais de l'émigration, soit par celui du renvoi dans le pays d'origine. Ce retour est marqué par différentes opérations d'une brutalité croissante. Dans le cadre des accords de paix, il s'opère tout d'abord via des rapatriements conjointement organisés par l'Ukraine et la Russie d'une part, et la Pologne de l'autre. Cependant, le rapatriement ne concerne qu'une frange réduite des Soviétiques présents sur le sol polonais, et aucunement ceux de Roumanie. Par une succession de décisions politiques et de décrets, la Pologne et la Roumanie optent alors pour l'expulsion, puis la déportation. La question des réfugiés vient cristalliser la lutte entre voisins pour le durcissement des frontières héritées de la guerre, concentrant certes les problèmes sociaux et humanitaires, mais soulevant aussi les enjeux politiques de l'après-guerre.

5 Néanmoins, le sort des réfugiés n'est pas réglé à leur retour en Ukraine. Leur arrivée pose la question d'une politique humanitaire aux premières heures de l'Union soviétique. Abandonnant aux organisations humanitaires - en l'occurrence, essentiellement le Joint pour les Juifs - le soin des réfugiés et des rapatriés, la politique d'accueil soviétique se concentre sur le parquement physique des retours et, paradoxalement, se caractérise par son côté policier. Pour autant, les institutions humanitaires ne parviendront jamais à piloter la gestion des réfugiés. Contrairement 
aux échanges de population qui se déroulent simultanément dans les Balkans et le cadre du conflit gréco-turc, ces retours soviétiques ne deviennent pas un problème international. Ils se limitent à des relations bilatérales dans lesquelles le Haut Commissariat, la Société des Nations et la Croix-Rouge jouent le plus souvent un rôle de spectateurs attentifs, et plus rarement de médiateurs. Ce cas soviétique constitue donc un exemple particulier de crise entièrement dominée par les États, dont les impératifs sont pour ainsi dire mis à nus, et où l'humanitaire ne peut se déployer que sous domination, sans jamais renvoyer aux argumentaires universalistes.

La focalisation sur les Juifs et sur ces allers et retours permet de complexifier l'appréhension du grand exil et des mouvements de populations qui ont accompagné la recomposition territoriale à l'est de l'Europe. Elle permet aussi de souligner la variété des acteurs autant que des trajectoires pour éviter le piège de la téléologie conduisant à la constitution d'une diaspora russe en exil. Le but est ici de mettre en lumière les interactions au sein des groupes de déplacés avec les différentes organisations humanitaires, mais aussi avec les institutions étatiques, qui ont toutes participé à infléchir l'avenir, dès lors privé de destin, des réfugiés ${ }^{3}$.

7 Les autorités manifestent une générale bienveillance à l'égard des Juifs poussés dehors par la Pologne ou la Roumanie, mais elles montrent aussi en sous-main une profonde défiance envers des citoyens trop longtemps restés à l'étranger. Ceux destinés ou décidés au retour demeurent, pendant toutes ces années, dans un entre-deux fait de lois d'exception, de décrets d'amnistie et d'impréparation technique. L'attention aux trajectoires heurtées des déplacés tentera ainsi de décrire avec précision les conditions de vie des réfugiés, généralement éclipsées par les enjeux institutionnels et les sources qui piègent l'analyse dans le point de vue de l'État, mais qui constituent pourtant une expérience comparable à celles de millions d'habitants des territoires recomposés d'Europe orientale.

8 L'historiographie récente a surtout démontré les continuités des catégories et des techniques d'encadrement des populations frontalières ${ }^{4}$. La séquence qui suit l'établissement des nouvelles frontières en Europe orientale est marquée par l'émergence de nouvelles techniques de gestion et de catégories de jugement et de classification sommaires pour les réfugiés et les minorités. Ces variations, ces bricolages et ces innovations mettent en lumière la politisation et l'instrumentalisation du «problème» des réfugiés, mais aussi le creuset qu'ont représenté les zones périphériques, non seulement en elles-mêmes comme marges, mais aussi dans une interaction avec les États voisins ${ }^{5}$. Il s'agit ainsi d'écrire dans le même temps une histoire de la restauration des États après les guerres mondiale et civile et des formes de gouvernement dans les territoires périphériques, par la marge du traitement des minorités, de leur définition et de leur stigmatisation, lesquelles renvoient en dernier ressort aux définitions que les États se font de leurs propres populations ${ }^{6}$. Par la marge par rapport aux attentions portées par les "États jardiniers", selon l'expression de Zygmunt Bauman, au cœur politique et ethnique de leurs citoyens et en suivant l'idée selon laquelle les sociétés homogènes et disciplinées rêvées se construisent autant par la promotion de groupes sociaux qu'en négatif par l'exclusion de groupes stigmatisés et par des interactions avec leurs voisins ${ }^{7}$. 


\section{Rapatriements et retours}

\section{Le temps des traités}

9 La présence de leurs ressortissants potentiels en terres étrangères est un enjeu majeur pour tous les acteurs des traités de paix qui suivent la révolution. La question du statut de ces étrangers, au premier rang desquels les prisonniers de guerre, et de leur rapatriement est envisagée dans les traités eux-mêmes. Il s'agit de donner une base légale à de futurs échanges de populations et de clarifier les conditions d'obtention des nouvelles nationalités issues de l'éclatement de l'Empire russe.

L'enjeu est prégnant dans les relations russo-ukraino-polonaises. La guerre soviéto-polonaise a conduit à l'internement de dizaines de milliers de prisonniers de guerre et la Pologne est devenue l'une des étapes majeures des réfugiés. Pour les Juifs fuyant les pogroms et les combats, la Pologne, qui faisait anciennement partie de leur zone de résidence imposée par le tsarisme, s'érige comme la destination première de leurs déplacements, sans considération pour les nouvelles frontières. La question revêt une place importante dans le traité de Riga signé le 18 mars 1921 entre la Pologne d'une part et la Russie et l'Ukraine soviétiques de l'autre - ces deux dernières rassemblées dans une délégation essentiellement russe, présidée par Adol'f Ioffe, l'un des artisans de la paix de Brest-Litovsk et des autres traités de paix du début des années 1920 avec les nouveaux voisins de l'URSS. Les retours prévus par le traité encadrent conjointement soldats internés et réfugiés. Priorité est censée être donnée aux prisonniers de guerre, mais le mouvement est double. Un article codifie précisément les populations envisagées, seules susceptibles de revendiquer la nationalité d'un pays sur lequel elles ne se trouvent pas :

[...] exilés et réfugiés doivent être entendus comme les personnes qui, avant le $1^{\text {er }}$ août 1914, résidaient dans l'une des parties contractantes, sont à présent sur le territoire de l'autre partie, et qui, durant la Guerre mondiale de 1914-1918, la guerre polono-russo-ukrainienne ou la guerre civile ont quitté les localités occupées ou menacées par l'ennemi, ou ont été évacuées sur ordre des autorités civiles ou militaires. Dans la catégorie des exilés ou des réfugiés sont de même inclus les anciens prisonniers de guerre capturés pendant la Guerre mondiale et qui, avant le $1^{\text {er }}$ août 1914, résidaient sur le territoire de l'une des parties contractantes et sont à présent sur le territoire de l'autre partie, ou, de même, les anciens soldats russes ou ukrainiens qui sont à présent sur le territoire de la République polonaise, à la condition de ne pas avoir été fait prisonnier par l'Armée polonaise régulière. ${ }^{8}$

Le point déterminant pour revendiquer la nationalité polonaise ou soviétique est donc la résidence avant le début de la Première Guerre mondiale.

\section{Le révélateur des prisonniers de guerre}

11 Cet apparent affichage du critère géographique est trompeur. Dans ses catégories et dans son traitement des populations originaires de la future Union soviétique, la Pologne introduit de nettes différences. À la faveur de la guerre soviéto-polonaise, dès 1920, la Pologne est la première à se crisper sur le traitement des Juifs ukrainiens présents sur son sol, soit comme réfugiés, soit comme prisonniers de guerre. Dès le début des hostilités, l'armée polonaise constitue une catégorisation complexe des prisonniers afin notamment d'isoler les communistes et les Juifs. Elle poursuit en cela le 
travail de distinction opéré lors de la Première Guerre mondiale par les empires multiethniques, qui internaient les prisonniers selon leurs origines, en particulier pour essayer de gagner leur faveur. La grille polonaise fonctionne en faveur de quelques nationalités, mais surtout en négatif, et au détriment d'autres groupes, singulièrement celui des Juifs. Des instructions du 3 septembre 1920 sont claires :

Les Juifs captifs sont à séparer, isoler et placer séparément. Ils doivent être divisés entre Juifs russes et Juifs polonais. En ce qui concerne les Juifs polonais ouvrir une enquête, afin de savoir s'ils sont soupçonnés de trahison ou de désertion et, dans le cas d'éléments de preuve, les envoyer à une cour martiale (spéciale). ${ }^{9}$

12 À l'inverse, les Polonais (ethniques) ne sont pas assujettis à une telle procédure, mais sont soumis à une propagande et un travail politique soutenus afin d'être gagnés à la cause nationale. Dans la pratique, cette séparation s'accompagne d'innombrables coups et exactions. Dans le camp de Novominsk, par exemple, un soldat de l'Armée rouge, qui est parvenu à s'échapper, témoigne, « ils ont choisi dans nos rangs les communistes, les commandants, les commissaires et les Juifs, et immédiatement, sous les yeux des soldats de l'Armée rouge, ils ont abattu un commissaire juif (je ne connais pas son nom ni son origine) $»^{10}$. Plus généralement, la Croix-Rouge sur place témoigne des mauvais traitements institutionnalisés à l'encontre des Juifs dans tous les camps polonais : « Les Juifs sont en général pieds nus ", enfermés toute la journée dans des baraquements surpeuplés et mal éclairés, dans des conditions d'hygiène sommaires ${ }^{11}$. La Croix-Rouge souligne aussi le fait qu'ils sont globalement internés avec les communistes, tous assimilés à des bolcheviks.

13 Le cliché antisémite du judéo-bolchevisme joue alors à plein. La Grande Guerre avait déjà constitué un moment de défiance à l'égard des populations juives. Cette défiance se radicalise profondément à la faveur de la guerre civile et de la guerre soviéto-polonaise. Les Juifs sont alors présentés comme des traîtres à la patrie polonaise, des déserteurs et des espions. La propagande les décrit systématiquement comme des partisans du bolchevisme et la participation, même très minoritaire, de Juifs au bref pouvoir soviétique en Pologne, achève de les condamner ${ }^{12}$.

\section{Les réfugiés comme Juifs}

14 La « question juive » est encore plus manifeste dans le traitement des réfugiés. Les Juifs constituent, de fait, la majorité des réfugiés massés aux frontières. Ils se distinguent notamment des chrétiens par leur statut et leur condition. Le traité de Riga est en effet venu sanctionner la distinction nette entre "prisonniers de guerre", " exilés " et "réfugiés ». Les premiers sont des soldats de la guerre soviéto-polonaise. Les deuxièmes sont essentiellement des partisans des armées antibolcheviques, souvent accompagnés par leur famille, réfugiés en Pologne. Soldats d'une guerre civile, partisans d'États déchus comme la république populaire d'Ukraine, ils sont essentiellement russes ou ukrainiens. Ils vivent dans des camps de réfugiés ouverts, où ils ont la possibilité de travailler. Certains tentent de monter des filières de recrutement pour relancer les armées antibolcheviques et sont par conséquent souvent qualifiés d'« internés ». La troisième catégorie, celle des réfugiés à proprement parler, regroupe des civils dispersés, répartis au gré des organismes d'aide ${ }^{13}$.

Parmi les Russes qui se sont réfugiés à l'étranger et plus particulièrement dans les pays limitrophes de la Russie, la proportion de Juifs est considérable.

À la détresse économique, à la famine, au chômage, aux multiples souffrances que 
toute la population de Russie avait endurées pendant ces dernières années, vint s'ajouter, pour les Juifs, une calamité terrible : les pogroms. Environ 150000 tués, 200000 mutilés, 50000 veuves, 170000 orphelins, un nombre considérable de familles ruinées, tel est, d'après les plus récentes estimations, le bilan tragique [...]. Dans les pays limitrophes de la Russie, notamment en Pologne et en Roumanie, ainsi que dans nombre de grandes villes de l'Europe occidentale, se trouvent actuellement groupés jusqu'à 150000 réfugiés juifs de Russie vivant dans une détresse indescriptible et sans possibilité de s'établir. ${ }^{14}$

institutions humanitaires juives. Le cas roumain semble le plus assuré. Les réfugiés sont
essentiellement massés en Bessarabie, ancienne région de l'Empire russe annexée par la Roumanie. La forte présence juive et la préexistence des institutions communautaires semblent favoriser le dénombrement des réfugiés et l'acheminement de l'aide. Début 1921, 24457 Juifs sont ainsi aidés par le Joint dans le pays; leur nombre a doublé en quelques semaines ${ }^{15}$. Cela laisserait supposer plus de 100000 Juifs réfugiés en Pologne. Les décomptes avancés par les différentes institutions sont contradictoires. Au gré des circonstances et des interlocuteurs, les chiffres sont tantôt grossis afin d'encourager l'aide, tantôt grandement minorés afin de laisser croire que la présence des Juifs est marginale ${ }^{16}$. Début 1923, le HCR présente toutefois une esquisse de la répartition des Juifs soviétiques en Pologne : $30 \%$ d'entre eux sont parvenus à rejoindre Varsovie, mais ils sont encore $65 \%$ à séjourner dans les « confins » frontaliers. Les derniers $5 \%$ sont répartis dans le reste du pays, en particulier dans la ville de Dantzig, dont le statut spécial et l'administration par la Société des Nations favorisent les possibilités d'émigration ${ }^{17}$.

\section{Les réfugiés comme problème}

Pour les deux États, roumain et polonais, l'accueil des «réfugiés russes » est perçu comme une charge sanitaire et économique. L'arrivée des milliers de réfugiés venus des zones de combat est instituée en problème public. Cette arrivée de migrants déguenillés est perçue comme une intrusion, voire comme une invasion. La situation est particulièrement tendue en Roumanie, dont la Bessarabie, tout juste annexée, est sous administration militaire. Les réfugiés manquent au premier chef de logements et sont confinés dans des synagogues et des propriétés privées, contaminées par les épidémies, tandis que les autorités refusent de louer au JDC ses bâtiments publics. Les Juifs ont interdiction de circuler et les regroupements sont placés sous la surveillance de soldats. Certains villages à majorité juive sont aussi transformés en « camp de concentration » pour y circonscrire les réfugiés ${ }^{18}$.

18 Dans ces États qui se rêvent homogènes ethniquement et religieusement, la question se focalise immédiatement sur les Juifs. Ces réfugiés sont pointés comme un vecteur de désordre incompatible avec l'affirmation d'un État souverain, qui plus est menacé par 
l'URSS. Chacun a quitté l'Ukraine en désordre, selon ses propres moyens, sans que nul convoi ne soit organisé ; cependant, ces arrivants sont présentés comme un groupe homogène et une menace sanitaire, économique et politique globale. Le premier ministre roumain parle de « péril juif » équivalent au « péril jaune » aux États-Unis ${ }^{19}$. Et tandis qu'il explicite son action lors d'un entretien, le Président du Conseil des ministres polonais, le général Sikorski, ne cache pas ses griefs à l'égard des réfugiés juifs :

une masse de Juifs passent chez nous par la frontière verte?

Par suite de l'immense longueur de notre frontière orientale, et de son insuffisante surveillance, l'envahissement de la Pologne a pris un caractère inquiétant, cela a attiré l'attention du gouvernement. [...]

L'État a dû porter son attention sur ces masses de réfugiés qui n'avaient pas droit d'asile et qui, par leur présence, menaçaient la vie économique et sociale du pays. On décida alors de soumettre ces réfugiés à un enregistrement rigoureux et à les obliger à quitter le pays dans la direction choisie par eux-mêmes. [...]

La guerre a forcé nos autorités à faire certaines concessions quant à la naturalisation polonaise. Il existe donc le danger prouvé par maints exemples que cet élément peu loyal, étranger à notre nation, profite de ces avantages pour s'établir sur le territoire de la République. ${ }^{20}$

L'accusation de déloyauté n'est pas neuve. La présence de Juifs de part et d'autre de la frontière avec l'Ukraine soviétique est supposée saper leur loyauté et risquer la souveraineté des États antibolcheviques. Les réfugiés sont ainsi accusés de colporter le bolchevisme. Ils sont porteurs d'une «virulente fièvre bolchevique » de la même façon qu'ils contractent le typhus - thème qui sera repris dans l'instauration du "cordon sanitaire $»^{21}$. Le Gouvernement polonais soupçonne même l'Ukraine soviétique d'avoir expulsé certains d'entre eux afin d'embarrasser le jeune État ${ }^{22}$. Dans tous les cas, les Juifs réfugiés ne peuvent être que les agents ou les jouets de l'étranger. En ce sens, les nouveaux voisins prennent à la lettre le mot d'ordre bolchevik d'effacement des frontières en faveur de la révolution mondiale ${ }^{23}$.

La Pologne et la Roumanie partagent avec l'Union soviétique une conception des minorités selon laquelle la force des liens ethniques de part et d'autre des frontières nationales permet d'envisager l'expansion du bolchevisme - ce que Terry Martin a qualifié de "principe du Piémont ${ }^{24}$. Ce principe est, au début de l'ère soviétique, un vecteur de déstabilisation régionale. Il est interprété de façon négative par les pays voisins, comme étant au fondement de la déloyauté des minorités frontalières, au premier rang desquelles les Juifs. La Pologne et la Roumanie sont, de fait, les premiers pays à placer ce principe à la base d'une gestion des populations qui prend rapidement un tour répressif.

21 Mais la présence des Juifs n'est pas seulement un problème extérieur. Elle est aussi une inquiétude économique. Les Juifs, réfugiés, pauvres et susceptibles d'accepter tout emploi même mal rémunéré, sont dénoncés comme une concurrence déloyale. Dans les campagnes où la révolution russe a chassé les anciens maitres, ils sont aussi une menace que relaye le délégué du HCR en Pologne :

il y avait chez les paysans une certaine méfiance à l'égard des réfugiés de toutes catégories; dans l'attente d'une réforme agraire, les paysans seraient, en ce moment, soupçonneux à l'égard de tous les gens qui, à leur avis, n'ont pas le droit de s'installer dans le pays; ils craignent de les voir participer aux avantages qui pourront leur être accordés. ${ }^{25}$ 
Que l'écrasante majorité des Juifs roumains et polonais soit intégrée dans les milieux du commerce, de l'artisanat et de la petite industrie n'y change rien.

\section{Premiers retours}

\section{États. Des commissions mixtes sont mises en place dans chaque pays, constituées, en} Ukraine, d'un représentant polonais, d'un membre du comité de rapatriement à proprement parler afin de régler les questions pratiques, et d'un membre de la police politique soviétique. Ces retours mêlent, dans de mêmes convois, prisonniers et réfugiés désireux de retrouver leur patrie d'origine. Les accords prévoient 4000 rapatriés par semaine, dont 1500 prisonniers de guerre ${ }^{26}$. Les rapports récapitulatifs sont toutefois contradictoires. Une première série établie par la commission soviétique de rapatriement fait état de 69000 soldats rapatriés pour à peine 9000 civils, Russie et Ukraine confondues. Cette seule dernière aurait vu le retour de 20000 prisonniers environ et de près de 2950 réfugiés ${ }^{27}$, tandis que la Pologne aurait récupéré pour sa part 20119 prisonniers et 191258 réfugiés $^{28}$. Un autre rapport du Haut Commissariat aux réfugiés transmis à la Société des Nations fait état de chiffres plus importants : 30398 prisonniers et 738023 réfugiés renvoyés en Pologne pour respectivement 72663 et 14286 vers les républiques soviétiques ${ }^{29}$. Si la commission semble plus certaine de ces premiers chiffres, le second rapport laisse entrevoir le peu de maîtrise des mouvements de populations des différentes parties en présence. La différence entre la Pologne et l'URSS est néanmoins très nette. Le Gouvernement polonais se plaint d'ailleurs à plusieurs reprises de cette disproportion entre civils et militaires, tandis que la délégation russo-ukrainienne insiste sur la lenteur des rapatriements vers la patrie soviétique ${ }^{30}$.

La vitesse avec laquelle les Soviétiques organisent les rapatriements s'apparente pour beaucoup à de la précipitation. Les Polonais prêts au retour sont en effet internés en Russie centrale qui, en cette fin d'année 1921, bascule dans la famine. Certains transferts, soumis aux difficultés matérielles d'un réseau ferré désorganisé, sont meurtriers. Les institutions internationales s'émeuvent notamment d'un convoi passant par Kazan' en mars 1922, et dont les deux tiers du contingent décèdent en route :

Un convoi de Kazan' a fait route pendant 90 jours. La distance entre Kazan' et la frontière polonaise est de 1663 verstes, soit 1739 kilomètres (environ 1100 miles). Le convoi parcourait $19,33 \mathrm{~km}$ en 24 heures, soit une moyenne d'environ $0,8 \mathrm{~km}$ à l'heure. [...] Il était composé de 1948 personnes, dont seulement 649 atteignirent la frontière polonaise. [...] La mortalité fut de 14,43 personnes par jour; en d'autres termes, un réfugié mourait toutes les heures et quarante minutes. Les morts étaient jetés en gare. ${ }^{31}$

Les retours s'étalent entre 1921 et 1923, mais l'ensemble des convois est parti lors de la première année. Le rapport de 1924 du HCR tente d'évaluer les retours par année. Selon lui, en 1921, 67203 prisonniers et 3534 réfugiés sont rapatriés vers les républiques soviétiques. En quelques mois, la plupart des camps de prisonniers de guerre sont donc liquidés, puisque seuls 5460 prisonniers sont rapatriés en 1922. Le rapport avec les réfugiés s'inverse alors nettement : ils sont 7715 à traverser la frontière soviétique en 1922, et encore 3037 en 1923 pour aucun prisonnier. Plus de $80 \%$ des retours ont donc été organisés en 1921 mais seul un quart des réfugiés est alors rentré32. 

soviétiques après la guerre, soit environ $10 \%$ des réfugiés présents en terres étrangères. La focalisation des traités de paix et des nouveaux États sur la question des retours ne doit pas occulter le mouvement général qui se joue alors au sein des réfugiés en Pologne, en Roumanie et plus généralement en Europe orientale, qui tend plus systématiquement à l'émigration qu'aux rapatriements. Les enquêtes auprès des réfugiés juifs aux frontières sont sur ce point sans appel: ils sont une écrasante majorité à chercher à émigrer, essentiellement aux États-Unis, et dans une très moindre mesure en Palestine et en Argentine ${ }^{33}$.

Toutefois, à l'arrivée, la composition des convois diffèrent largement de ce qu'elle était au départ. Des retours spontanés viennent s'y greffer, ajoutant de nouveaux réfugiés aux rapatriés. Les données recueillies par le Commissariat du peuple aux Affaires étrangères (NKID) ukrainien accusent ainsi un net écart lors de l'entrée sur le territoire soviétique. Pour le seul mois de juillet 1921, 3591 soldats sont rapatriés des camps d'internement vers l'Ukraine, et 3189 réfugiés $^{34}$, soit plus de réfugiés en un mois que le total enregistré sur deux ans par la commission de rapatriement ! La fin de la guerre civile et la pression mise sur les réfugiés entraînent des retours spontanés au moins aussi importants que les rapatriements, dont les convois servent ainsi de déclencheurs, d'appels d'air. Confronté à ce phénomène, le Commissariat du peuple aux Affaires intérieures (NKVD) demeure impuissant et fond les deux types de déplacement ${ }^{35}$.

\section{Expulsions et déportations}

\section{Vers les expulsions}

Le règlement de la question des réfugiés échappe largement au mouvement des retours organisés, surtout après la liquidation des camps de prisonniers de guerre. Les rapatriements, où les partants sont volontaires, ne touchent qu'une population limitée. Les réfugiés venus d'Ukraine sont peu tentés de retourner dans une région encore en proie aux troubles et aux pénuries alimentaires. Enfin, les organismes d'aide aux Juifs soulignent que pendant toute la période 1920-1924, les réfugiés demeurent peu ou pas au courant des initiatives étatiques dont ils n'ont connaissance que sur le tard.

En Pologne, l'opposition «conservatrice» accuse le gouvernement de laisser les frontières poreuses et d'entretenir un État faible dans ses marges ${ }^{36}$. Après les rapatriements, la présence de réfugiés étrangers continue à symboliser la faiblesse des nouvelles frontières, d'autant que de nouveaux déplacés continuent d'arriver, bien que dans une moindre mesure. En avril 1923, un télégramme de Sikorski, le Président du Conseil des ministres polonais, résume son sentiment après 1921 :

En outre le passage illégal de notre vaste frontière orientale s'est poursuivi. Elle est ouverte et difficile à surveiller. Cet état de choses inadmissible portant atteinte à la souveraineté de la Pologne a attiré l'attention du Gouvernement et de la Diète de Pologne qui ont demandé en mars dernier de prendre les mesures les plus sévères pour remédier au mal [...] Le Gouvernement continue de recevoir hospitalièrement et d'héberger tous les réfugiés politiques et religieux venant de Russie bolcheviste mais ne saurait consentir à ce que ses frontières orientales soient ouvertes ni que le séjour des réfugiés déjà arrivés dure indéfiniment. ${ }^{37}$

Une série de mesures, en Pologne et en Roumanie, tente de consolider la souveraineté aux frontières. La frontière polonaise est officiellement fermée le $1^{\mathrm{er}}$ juillet 1921 et 
toute pénétration sur le territoire est donc frappée d'illégalitée ${ }^{38}$. Dans le même temps, en mai en Roumanie et en juin en Pologne, ordre est donné aux réfugiés de quitter les zones frontalières. L'armée roumaine l'applique sur une bande de dix verstes (un peu plus de dix kilomètres) le long de la frontière avec l'Ukraine, suivant en cela l'exemple soviétique; la Pologne utilise le découpage administratif ${ }^{39}$. Ces mesures s'intègrent au souci général d'instauration de zones tampons à la sécurité renforcée. L'évacuation n'est qu'un des aspects de cette prise de contrôle totale de territoires jugés stratégiques ${ }^{40}$.

Dans le but de contrôler aussi les mouvements de population, le pouvoir polonais tente d'imposer un enregistrement obligatoire, auprès des antennes de police locales, des réfugiés sur son territoire ${ }^{41}$. Le but semble surtout de s'assurer que les réfugiés organisent leur émigration, mais dans cette optique, ceux-ci préfèrent très largement se tourner vers les organismes d'aide juifs qui assurent tant le financement que les démarches auprès des autorités compétentes, à commencer par les antennes diplomatiques. Les réfugiés juifs continuent à largement se soustraire à l'État. Ils n'ont en effet aucune raison de se déclarer auprès des autorités afin de bénéficier des statuts de réfugié politique mis en place après la guerre et qui se limitent essentiellement en Pologne aux partisans des armées antibolcheviques ${ }^{42}$.

\section{Premières restrictions}

31 Les premières mesures visant à sécuriser la frontière en juin 1921 entraînent les premières expulsions de réfugiés présents dans les villes frontalières. Pendant tout l'été, dans les régions polonaises de Galicie et de Volhynie, des opérations visent notamment les lieux de regroupement et d'aide aux réfugiés. Ceux qui ne sont pas en règle ou qui ne peuvent démontrer leur imminente émigration sont contraints à la déportation vers l'Ukraine soviétique. À Podwoloczysk, en Galicie, entre 200 et 250 Juifs sont ainsi arrêtés, et 66 expulsés en juillet. En août, c'est surtout la Volhynie qui est visée. Durant ces premières expulsions, note un rapport du Joint, certains Juifs ayant droit à la nationalité polonaise et tout juste rapatriés par les Soviétiques, mais n'ayant pas encore obtenu leurs documents, sont parfois expulsés. Dans le chaos des retours d'après-guerre, toute irrégularité peut valoir prétexte d'expulsion. Les Juifs ukrainiens à Varsovie sont aussi visés en septembre. Une liste de 2587 réfugiés est affichée en ville, avec ordre de départ immédiat. Afin d'appliquer ces mesures, des contrôles systématiques de police sont opérés dans les gares de Varsovie et des grandes villes des régions frontalières. Les expulsions se poursuivent en Galicie, en particulier à Lwów, jusqu'en janvier 1922, afin d'en refouler tous les réfugiés ${ }^{43}$.

Contrairement aux mesures polonaises, les ordres d'évacuation roumains de mai 1921 laissent la possibilité aux réfugiés, sous contrôle $\mathrm{du} J \mathrm{JC}$, de se replier vers l'intérieur $\mathrm{du}$ territoire. Les antennes des organismes humanitaires sont liquidées et ces nouveaux déplacements sont l'occasion d'arrestations et d'expulsions vers l'Ukraine, touchant une fois encore des Juifs susceptibles de fournir des documents d'émigration en règle. Une nouvelle évacuation est ordonnée en novembre, avec le même résultat. À Chisinau (ex-Kichinev), les rumeurs n'évoquent pour commencer que quelques wagons à destination de l'Ukraine, concernant quelques dizaines de personnes. Plus encadrés qu'en Pologne, les réfugiés juifs font les frais de cette situation. Au final, les autorités militaires imposent la déportation de 1400 Juifs, à charge aux organismes humanitaires 
de les rassembler. Ces derniers soumettent une liste de 981 noms. Seule l'intervention in extremis des organisations juives soutenues par la Croix-Rouge et le Dr Nansen en personne, les sauve de la déportation vers une Ukraine méridionale ravagée par la famine. De nouveau, le déplacement vers l'intérieur de la Bessarabie roumaine est privilégié $^{44}$.

Une seconde étape dans le traitement des réfugiés polonais intervient en mars 1922. Le 17, les autorités de Rovno imposent l'enregistrement systématique des réfugiés arrivés après le 12 octobre 1920, sous peine d'expulsion. Les autorités polonaises ne cherchent plus alors seulement à sécuriser une zone frontalière, mais à encadrer une population jugée comme problématique depuis des années. La date butoir d'enregistrement est le $1^{\mathrm{er}}$ avril, à peine deux semaines après la publication de l'ordre auquel de nombreux réfugiés n'ont pas le temps de se soumettre. Des raids réguliers de la police entraînent de nouvelles expulsions. Le même scénario se déroule à Lwów où les réfugiés qui ne se sont pas enregistrés sont expulsés au-delà du Zbru@, vers l'Ukraine soviétique, sans avoir eu le temps de rassembler leurs affaires personnelles. Pendant la première moitié de l'année 1922, ces enregistrements et ces expulsions s'accompagnent de la fermeture forcée de centres d'aide aux déplacés juifs afin, selon les autorités, de ne plus attirer de réfugiés et, pratiquement, d'en arrêter l'arrivée de nouveaux ${ }^{45}$. Dans cette seconde étape, les maires (starostes) et les autorités régionales ont joué un rôle moteur, parfois même en contradiction avec les initiatives nationales, notamment du ministère de l'Intérieur polonais, soumis aux pressions des organisations internationales.

\section{Les décrets d'expulsion}

Après le nettoyage des frontières, la Pologne et la Roumanie envisagent de régler définitivement la question des réfugiés sur leur sol. Le but affiché est le départ de l'ensemble des réfugiés juifs qui n'ont pas vocation à demeurer dans ces pays d'accueil. Déjà en juillet 1921, la Pologne avait répondu à une première suspension des départs à destination des États-Unis par une série de menaces d'expulsion ${ }^{46}$. Les réfugiés, représentés comme une menace intérieure, servent aussi de moyen d'affirmation d'une souveraineté sur la scène internationale. Par les expulsions, la Pologne tente de faire des réfugiés un problème international, mais le nouveau gouvernement cherche aussi à en partager régulièrement le coût avec les pays voisins, à travers la Société des Nations et des demandes de financement international. Le problème devient aigu à la fin 1922, deux ans après la paix signée entre la Pologne et les pays soviétiques, et un an après les premières reconduites à la frontière. Les deux pays se plaignent tout au long de l'année auprès du Haut Commissariat aux réfugiés de la charge économique et sanitaire représentée par les réfugiés et de la limitation des quotas américains, atteints prématurément ${ }^{47}$.

35 L'outil privilégié afin de mettre un terme à leur présence prend la forme, en Pologne et en Roumanie, de décrets intimant l'ordre aux réfugiés de prendre des dispositions précises (passant essentiellement par l'obtention d'un visa) en vue d'émigrer sous peine d'être expulsé vers le pays d'origine présumé : l'Union soviétique. Dans les faits, les décrets permettent surtout aux autorités de se défaire de la charge des réfugiés en les repoussant au-delà de leurs frontières, précipitant les départs ou les expulsions. Le ministre de l'Intérieur polonais signe le 5 décembre 1922 un décret ordonnant l'expulsion de tous les réfugiés qui n'auront pas quitté le pays avant le $1^{\text {er }}$ mars 1923. La 
pression des organisations juives polonaises et internationales, et surtout du Haut Commissariat aux réfugiés auprès de la Société des Nations, repousse de quelques semaines le délai, sans pour autant faire fléchir le gouvernement. Un mémorandum du HCR résume alors la situation :

Le ministère de l'Intérieur de la République polonaise a décrété de nouvelles mesures à l'égard des réfugiés russes entrés illégalement en Pologne depuis le 12 octobre 1920. Ces mesures ont paru dans une circulaire adressée à tous les Wojevoda [voïvodies], le 9.II.1923. [...]

Les réfugiés non enregistrés seront expulsés d'ici au 15 avril 1923. Les réfugiés enregistrés seront expulsés à partir du 15 avril. ${ }^{48}$

$\mathrm{Au}$ printemps 1923, la Roumanie établit un décret identique dans "l'intention d'évacuer de Bessarabie en Russie, pour des raisons militaires, environ 10000 réfugiés"; tandis que la Pologne ne fait que retarder son application, le gouvernement roumain prend le parti de suivre les insistantes demandes de Nansen et du HCR, et renonce un temps aux expulsions ${ }^{49}$.

Les décrets sèment la panique parmi les réfugiés. Leur annonce conduit 1000 à 1500 Juifs réfugiés en Pologne à chercher à rejoindre la ville de Dantzig, dont le statut spécial les protégerait des rafles ${ }^{50}$. Malgré le report des expulsions jusqu'au 15 avril 1923, les responsables locaux entreprennent immédiatement de déporter des réfugiés vers la frontière. De fait, la modération gouvernementale semble surtout s'adresser aux organisations internationales. L'envoyé de Nansen assure que dans les ministères «les lois d'expulsion ne sont et ne seront pas appliquées rigoureusement " si les réfugiés continuent à quitter d'eux-mêmes la Pologne ${ }^{51}$. La tonalité est différente à l'intention des responsables locaux. Pour le général Sikorski, «les autorités [...] sont appelées à exécuter sans appel ma décision » d'expulser les réfugiés qui ne sont pas en règle ${ }^{52}$.

Ainsi en Galicie, 8000 réfugiés sont reconduits vers l'Ukraine en février ${ }^{53}$. À Korec, en Volhynie, le staroste prend prétexte du décret pour expulser 25 familles juives, normalement épargnées car arrivées avant octobre 1920; ailleurs, 200 à 300 Juifs rapatriés, susceptibles de recevoir la nationalité polonaise, sont expulsés malgré les protestations ${ }^{54}$. Comme en 1922, les autorités locales jouent un rôle clef dans l'application brutale des décrets, voire dans l'extension de leur cible. Le Haut Commissariat aux réfugiés, en lien avec les seules autorités nationales, ne compile en effet que 1200 Juifs frappés d'expulsion en Pologne après le 15 avril, malgré un phénomène plus ample ${ }^{55}$.

La politique roumaine s'infléchit plus tardivement. Bucarest prend en septembre 1923 le parti de modifier sa politique, jusque-là plus conciliante qu'en Pologne. Les expulsions reprennent afin de liquider la question des réfugiés en Bessarabie. Cependant, contrairement à la Pologne qui les conduit systématiquement à la frontière, la Roumanie tente de nouveau de déléguer le problème à des organismes juifs, comme la Jewish Colonisation Association, qui prend en charge 1000 réfugiés afin de les réinstaller en Ukraine ${ }^{56}$.

Les Juifs jugés illégaux ou raflés lors des grandes opérations de police sont reconduits à la frontière manu militari et déportés de force en URSS. Le chaos et la menace pèsent lors des expulsions que décrivent et dénoncent tant les organismes d'aide juifs que les observateurs internationaux de la Croix-Rouge ou de la Société des Nations. En Pologne ou en Roumanie, la mise en place est semblable. Dès le mois de février 1923, des députés juifs polonais tentent d'alerter le gouvernement sur le processus enclenché : 
L'expulsion se fait de la manière suivante : les chariots, chargés de vieillards, de femmes et d'enfants, sont reconduits par la police à cheval jusqu'à la frontière que les réfugiés franchissent sous la menace de fusils, pour voir, du côté bolcheviste d'autres fusils braqués sur eux; de sorte que les victimes de l'expulsion errent, transies de froid, entre les deux lignes de postes frontières. ${ }^{57}$

41 En ce début d'année 1923, les anciens habitants de l'Ukraine réfugiés dans les pays limitrophes ont en effet perdu leur droit à la citoyenneté soviétique. Par décret, le pouvoir soviétique a déchu de leurs nationalités, le 15 décembre 1921, les personnes dépourvues de passeport soviétique, de même que celles qui ont quitté le pays avant la date d'entrée en vigueur du décret, si elles ne se font pas enregistrer dans les trois mois et demi ${ }^{58}$.

Peu au fait des dispositions légales et plus globalement craintifs à l'égard de tout enregistrement, les Juifs d'Ukraine réfugiés sont donc pour un temps apatrides et aucun État ne semble enclin à les accueillir. Les organisations juives occidentales d'aide à leurs coreligionnaires d'Europe orientale, s'alarment d'une situation sans issue :

La plupart [des réfugiés] ayant quitté leur pays sans l'autorisation des autorités soviétiques, celles-ci ne les reconnaissent plus comme des ressortissants russes, leur refusant impitoyablement leur rentrée dans leur patrie. L'on signale déjà des cas de ces réfugiés qui, reconduits à la frontière par des gardes polonais, ont été également repoussés par les sentinelles rouges. ${ }^{59}$

Le problème est résolu par les autorités soviétiques le 13 avril, via une amnistie générale des réfugiés présents en territoire polonais, à condition de se présenter avant le $1^{\mathrm{er}}$ juin à la légation soviétique en Pologne à Varsovie. Dans les faits, l'amnistie leur rouvre le droit à la citoyenneté soviétique à condition de faire la preuve qu'ils sont bien originaires d'Ukraine et qu'ils y ont résidé jusqu'en $1914^{60}$. Certains expulsés ont donc passé plusieurs semaines, immobilisés en zone neutre, pris entre les deux feux des armées polonaise et rouge. L'amnistie est aussi l'occasion pour « un grand nombre de réfugiés juifs Ukrainiens, sous le coup du décret d'expulsion » d'entamer un retour à leurs propres frais et de s'insérer dans les retours forcés, comme auparavant dans les rapatriements $^{61}$. De 1921 à 1924 , le mouvement est toujours double, combinant retours encadrés par les rapatriements ou les déportations, et retours spontanés.

\section{Concentration et filtration}

\section{Impréparation soviétique et humanitarisme juif}

Malgré les accords et les amnisties, l'encadrement des réfugiés et de leur retour se révèle une tâche très difficile pour Kiev. Avant la création formelle de l'Union soviétique en décembre 1922, les négociations tripartites, essentiellement menées par la Russie, compliquent la gestion ukrainienne. Les retours spontanés se font très largement en dehors de tout contrôle étatique. Le Commissariat du peuple aux Affaires intérieures (NKVD) envoie perpétuellement des demandes de renseignements à ses antennes locales et aux autres administrations, afin de cerner le problème. Le 23 décembre 1921, un télégramme de Kiev se réduit à demander : " prière d'informer rapidement sur la question des réévacués et des réfugiés ${ }^{62}$. La brièveté des requêtes laisse entrevoir la méconnaissance complète des développements sur le terrain, dans des régions tout juste administrées par le régime soviétique et mal gardées ${ }^{63}$. Les 
brusques à-coups des politiques polonaise et roumaine entravent toute concertation et obligent l'Ukraine soviétique à gérer les réfugiés dans la précipitation.

Les expulsions étaient menées avec une grande brutalité, les réfugiés étant poussés par des forces armées au-delà de la frontière russe où ils étaient arrêtés par les autorités russes et envoyés en prison jusqu'à ce que leur cas soit investigué par le Département politique [le GPU, la police politique]. ${ }^{64}$

Face à l'afflux de déplacés, préférence est donnée aux « camps de concentration » pour réfugiés, situés dans des villes frontalières. Les déplacés de Pologne sont accueillis dans le camp de Chepetovka et ceux de Roumanie dans celui de Novograd-Volynsk, après une étape par la ville de Kamenetz-Podolsk. Plusieurs «étapes » sont en effet installées le long de la frontière afin de rediriger les réfugiés vers les camps, qui servent surtout de camps de transit avant le transfert à Kiev où une enquête plus approfondie est entreprise. Lors de leur transit, certains réfugiés sont aussi retenus un temps à la prison de Žitomir, où 400 d'entre eux furent incarcérés.

Le contrôle est exercé par les autorités soviétiques, mais l'accompagnement des réfugiés est assuré par les organismes humanitaires juifs, au premier rang desquels le Joint Distribution Committee. Celui-ci s'appuie aussi sur les institutions et responsables juifs ukrainiens préexistants, singulièrement le Comité d'aide aux victimes juives des pogroms (Evobkom), qui a fédéré toutes les organisations juives d'aide aux victimes de la guerre. Le Joint assure l'approvisionnement des rapatriés : «la ration journalière consiste en : 1,5 livre de pain, 10 onces de sucre et 2 œufs. Les enfants reçoivent du lait ", ce qui, dans une Ukraine qui se relève tout juste de la famine de 1921-1922, représente un régime alimentaire solide et mobilise des sommes considérables à l'échelle ukrainienne. Dans les faits, et en dépit de l'encadrement carcéral, la situation des prisonniers à Žitomir n'est pas très différente. Là aussi, le JDC pourvoit à la nourriture et accompagne les réfugiés en attente de leur envoi à Kiev et d'une enquête plus approfondie ${ }^{65}$.

47 L'aide alimentaire est aussi favorisée par l'apport des cantines d'aide aux victimes de la famine qui poursuivent leur mission et, en 1923, combinent aide aux affamés et aide aux réfugiés. Le Joint avait en effet déjà repris pied en Ukraine en 1921 par suite de la famine. Un accord passé avec l'American Relief Association (ARA), alors pierre angulaire de l'aide alimentaire, avait permis d'acheminer des fonds vers l'Ukraine soviétique. L'accord permettait au Joint d'intégrer les missions et les cantines de l'ARA et prévoyait même la constitution d'un circuit d'aide parallèle entièrement géré par l'organisme juif. Ce dernier pan du projet ne put être accompli, mais en 1922 le Joint gérait néanmoins de nombreuses cantines, notamment près de la moitié de celles destinées aux enfants, sans distinction de nationalité. En Pologne et en Roumanie, où il était actif dès 1919, il accueillait aussi de nombreux réfugiés de la famine, s'appuyant en particulier sur les Landsmanschaften, ces sociétés d'aide financées par les Juifs originaires de la ville et émigrés, qui maillaient une grande part de l'ancienne zone de résidence tsariste ${ }^{66}$.

48 Le camp de Kiev abrite ainsi, en février 1923, 600 internés. Le pic est atteint entre juin et août quand, par suite des déportations, 1300 Juifs y sont internés. Matériellement, lors de la guerre civile, le camp est un ancien camp de concentration pour suspects tenu par la police politique, la čéka, l'un des maillons de la répression politique. Cependant, en 1923, une autre fonction lui est attribuée. Le JDC l'administre de facto 
mais la direction demeure aux mains des autorités soviétiques. Avec cette cogestion, les réfugiés bénéficient d'une certaine liberté :

À Kiev, nous avons organisé une cuisine dans le camp où la nourriture est distribuée aux réfugiés, avec une ration de 2 livres de pain par jour, d'une soupe chaude le matin, d'une soupe avec de la viande à midi et du thé le soir. Nous réparons actuellement les bains et désinfectons les baraques et leurs vêtements.

Le Gouvernement traite très bien les réfugiés. Ils ont une absolue liberté et on prend soin d'eux de toutes les manières possibles. Le directeur du camp est intéressé par leur bien-être. ${ }^{67}$

Le rapport étant à usage interne du Joint, il est peu probable que les louanges soient feintes. Elles sont d'ailleurs reprises dans le rapport plus général établi à propos de l'activité du Joint pendant ces années, même si elles valent surtout par contraste avec les mauvais traitements subis en Pologne ou en Roumanie : à titre de comparaison, les réfugiés internés en Pologne n'avaient droit qu'à une demi-livre de pain ${ }^{68}$.

Le Joint assure aussi les soins médicaux et jusqu'aux transferts de camp à camp. Il prend à sa charge les frais de déplacement et obtient du Commissariat du peuple aux Transports une réduction de $50 \%$ sur les tarifs en vigueur. L'accord permet ainsi aux rapatriés de bénéficier d'un transport dans des conditions optimales, après les convois catastrophiques des rapatriements précédents. Le représentant du Joint peut conclure son rapport en soulignant que les réfugiés sont pris en charge « depuis la minute où ils passent la frontière jusqu'à ce qu'ils soient effectivement libres $»^{69}$.

Le pouvoir soviétique ne souhaite néanmoins pas abandonner la gestion des mouvements de population à des organismes privés et étrangers. Une fois les rapatriements organisés achevés et les expulsions amorcées, le NKID ukrainien tente de canaliser le nouvel afflux de réfugiés juifs en Ukraine. Il met ainsi en place à la fin de l'année 1923 une "Société pour le rapatriement des réfugiés juifs » dont la logistique est assurée par le Haut Commissariat aux réfugiés. Son but est donc essentiellement de (re) faire de la gestion des réfugiés une affaire étatique ukrainienne, et non plus humanitaire. À sa création, la société prévoit surtout de rapatrier un millier de réfugiés de Roumanie. Toutefois, dans la masse des mouvements de population, l'initiative soviétique semble intervenir trop tard pour réellement encadrer les retours globalement assurés par le Joint ${ }^{70}$.

\section{"Quarantaine politique » et opérations de contrôle}

52 Si le contrôle soviétique sur les réfugiés retournés sur le sol ukrainien ne parvient pas à se faire en amont de leur départ, il s'opère surtout en aval, à l'entrée sur le territoire, à travers une enquête administrative. Le soupçon plane sur ces populations déplacées par la guerre. Selon une note du dirigeant de la police politique et du NKVD russe puis soviétique, Feliks Dzeržinskij, il s'agit surtout d'appliquer, dès le 21 avril 1921, soit dès les premiers rapatriements, "un système de quarantaine politique à tous les contingents rentrant en Russie ou en Ukraine ", réfugiés ou prisonniers de guerre, "seule garantie contre les éléments politiquement dangereux " susceptibles d'entrer sur le territoire soviétique ${ }^{71}$.

De même que le pouvoir soviétique a entrepris un travail de propagande auprès des prisonniers de guerre polonais pour les gagner à sa cause, il craint que les individus de retour sur son territoire ne soient soumis à l'influence des pays ennemis. Non sans raison, puisque, encore en 1921, une "agitation antisoviétique » est menée dans les 
camps de prisonniers de guerre, notamment en faveur du recrutement pour les armées antibolcheviques russes ${ }^{72}$. La délégation ukraino-russe en charge des rapatriements prend ainsi soin d'exiger que les rapatriés ne soient en aucun cas mis en contact avec des " éléments antisoviétiques " $^{73}$. Entre la quarantaine et la contagion, le lexique qui prévaut alors au sein des autorités politiques est celui de la maladie qu'il s'agit d'endiguer afin d'épargner le jeune corps politique soviétique, déjà miné par les contestations lors de la guerre civile.

Le travail d'enquête est assigné à la police politique à travers son antenne au sein du NKVD. L'investigation revêt ainsi la forme d'une banale opération de police au sein d'une "commission de filtrage». Le NKVD est de fait le seul organisme susceptible d'investiguer autant de réfugiés à un moment, la fin de la guerre civile, où les effectifs de la police politique on été rapidement réduits. Le bricolage institutionnel de l'opération de filtrage - mêlant humanitaire, routine policière et police politique témoigne aussi du désir de parer au plus pressé dans une situation de carence bureaucratique.

Les documents de cette commission reflètent des enquêtes de routine qui visent essentiellement à préciser ou à confirmer auprès des autorités locales les éléments biographiques allégués par le rapatrié. Pour celui-ci, le but est surtout de démontrer son bon droit à la citoyenneté soviétique, malgré les mois passés à l'étranger ${ }^{74}$. En effet, l'enquête mentionne aussi la date de départ des réfugiés : la plupart ont fui l'Ukraine en 1919, au pire de la persécution pogromiste et de la guerre civile, mais dans le même mouvement sont aussi rapatriés quelques réfugiés de la Première Guerre mondiale, déplacés en 1915 ou par suite de la famine de 1921-1922. Si les victimes de 1919 constituent le contingent le plus important, d'autres ont passé près de huit ans sur les routes, ou au contraire à peine quelques mois. Selon le Joint, les vérifications prennent en général une douzaine de jours, "et lorsque quelqu'un était malade», le GPU « examinait le cas le plus rapidement possible " $^{75}$.

Le soupçon à l'égard des retours ne provient pas seulement du possible endoctrinement des réfugiés par la propagande étrangère. La campagne de rapatriement discerne trois catégories de réfugiés, forgées, c'est notable, par la section juive du parti communiste (Evsekcija) dès 1920 afin de trier les réfugiés présents en territoire soviétique. Reprises par l'État soviétique dans son ensemble et par la commission de rapatriement en particulier, ces catégories distinguent: les «ex-capitalistes et propriétaires qui ne souhaitent pas adopter les modes de vie et de travail soviétiques", les réfugiés de guerre qui ont fui les combats et ceux qui souhaitent se rapprocher de leur famille (maris et femmes séparées par la guerre ou plus souvent, proches parents ayant besoin d'être entretenus pour cause de maladie ou d'invalidité $)^{76}$. Comme les opposants politiques déclarés, la première catégorie est aussi exclue du droit à la citoyenneté soviétique. Cependant, dans l'ensemble, les Juifs réfugiés ne sont pas inquiétés, selon le Joint, « en dépit du fait que les réfugiés de Pologne et de Roumanie ont formellement fui le Gouvernement soviétique et, plus généralement, que leurs anciennes positions sociales ne contribuaient pas à les faire considérer comme des éléments désirables dans un État socialiste $»^{77}$.

57 Après étude de leur cas, les réfugiés reçoivent des tickets de transport gratuits visés par le NKVD et délivrés par le GPU, mais seulement grâce au financement du JDC. Deux destinations sont possibles: Harkov' ou Odessa ${ }^{78}$. Pour les autres, le retour semble se faire par leurs propres moyens. 


\section{Conclusion}

Ballottés entre la pression des pays d'accueil et la suspicion soviétique, les réfugiés juifs venus d'Ukraine révèlent plusieurs logiques d'appréhension des minorités nationales forme de face-à-face qui augmenta les tensions sur la question des réfugiés et permit de les utiliser à des fins politiques d'affirmation d'un corps social, national et ethnique, et diplomatiques d'affirmation de la souveraineté. Le soupçon perpétuel du judéo-bolchevisme, porté tant par l'État roumain que l'État polonais, notamment par leurs armées, tend à stigmatiser la population juive dans sa totalité comme une minorité agissant au seul profit de l'Union soviétique. L'URSS n'est pas le seul État à craindre perpétuellement la porosité de ses frontières et l'infiltration du pays par des minorités organisées politiquement. De ce point de vue, les grandes opérations de «nettoyage » et de "sécurisation » des frontières opérées dans les années 1930 par le pouvoir stalinien se caractérisent par leur importance et leur systématicité mais pas par leur conception des populations aux frontières qui participent d'un cadre est-européen plus large ${ }^{79}$. La place disproportionnée donnée au petit contingent de rapatriés et de déportés souligne toutefois que la radicalisation des États polonais et roumain dans le traitement de leurs propres minorités juives n'est pas seulement une histoire interne et autocentrée, elle renvoie à des interactions interétatiques. Juifs et soviétiques, ces déplacés sont venus incarner concrètement la menace fantasmée du judéo-bolchevisme. Les Juifs de l'ancien Empire russe ont ainsi collectivement pâti du redécoupage des frontières et des déplacements de population qui, par suite de la guerre, sont venus déchirer l'ancienne zone de résidence.

Le rôle matriciel de ces déplacements forcés de population vaut notamment en Roumanie. Les régions touchées par les expulsions connaissent une nouvelle crise lors du pacte germano-soviétique de 1939. L'annexion de la Bessarabie par l'URSS remet à vif la crainte d'une déloyauté des Juifs, accusés de préparer une invasion soviétique. La première phase de la persécution des Juifs de Roumanie est marquée en 1941 par une série de déportations. Dès le 22 juin, l'armée roumaine projetait de «nettoyer le terrain » des zones frontalières et, rapidement, de combiner massacres et déplacements forcés afin de liquider une population juive jugée complice des bolcheviks et néfaste à l'effort de guerre ${ }^{80}$. L'extermination est encore loin en 1921. Toutefois, la gestion des réfugiés juifs témoigne de l'institution rapide, à partir de la Première Guerre mondiale, des minorités juives transfrontalières comme problématiques et menaçantes à chaque fragilisation des frontières.

Une forme de continuité s'opère aussi du côté soviétique. Le phénomène de filtration se retrouve notamment lors du rapatriement des travailleurs déplacés par les Allemands vers l'Europe centrale, en particulier depuis l'Ukraine, pendant la Seconde Guerre mondiale. Parfois volontaires mais surtout victimes civiles de la guerre, ces Ostarbeiter sont massivement internés en camps. Le phénomène de filtration ne prend pas alors la forme d'une banale opération de police, il sert très largement à stigmatiser et à réprimer des éléments jugés déloyaux et collaborateurs. La filtration soviétique met ainsi en exergue les éléments personnels des personnes considérées sur une base de suspicion individuelle. À l'inverse, la filtration polonaise prend immédiatement pour base une suspicion généralisée et une forme de culpabilité collective, sur une base 
nationale. Elle vise un groupe infiltré dans la masse des "réfugiés russes »: les Juifs, constitués en une sorte de groupe parasite et irréductible, presque surnuméraire dans la nouvelle carte des populations d'Europe centrale et orientale.

61 Une même organisation prévaut dans le cas des échanges de population entre la Pologne et l'Ukraine après 1945 , visant à homogénéiser ethniquement les deux pays, à travers plusieurs phases et dans une brutalité croissante, et à effacer ces zones frontalières multiethniques afin de consolider définitivement les frontières d'après-guerre. Comme après 1920, des commissions mixtes polono-soviétiques opèrent dans un cadre bilatéral. Dans des proportions bien supérieures - plus d'un million de déplacés -, les déplacements s'effectuent de nouveau dans des conditions déplorables. Les partants sont dans un deuxième temps chassés par l'armée polonaise, venue à la rescousse d'une entreprise de déplacement d'une population privée de moyens par la guerre finissante ${ }^{81}$.

\section{NOTES}

1. Je remercie Alain Blum et Catherine Gousseff pour leurs conseils et lectures tout au long de ce travail. Ma gratitude va aussi aux relecteurs anonymes qui ont aidé à faire de ce travail de thèse un article à part entière.

2. Russo-Jewish Refugees in Eastern Europe, Londres: Offices in the Joint Foreign Committee, 1921 , p. 20.

3. Une « histoire interne de l'émigration ", pour reprendre l'expression de Catherine Gousseff, L'exil russe : La fabrique du réfugié apatride (1920-1939), P. : CNRS, 2008, p. 12.

4. Peter Holquist, Making War, Forging Revolution: Russia Continuum of Crisis, 1914-1921, Cambridge : Harvard University Press, 2002 ; Juliette Cadiot, Le laboratoire impérial : Russie-URSS 1860-1940, P. : CNRS, 2007.

5. Sabine Dullin, La frontière épaisse : Aux origines des politiques soviétiques (1920-1940), P.: EHESS, 2014.

6. Nick Baron, Peter Gatrell, « Population Displacement, State-Building, and Social Identity in the Lands of the Former Russian Empire, 1917-1923 ", Kritika, 4, 1 (Hiver 2003), p. 51-100.

7. Approche par le centre notamment déployée par István Deák, « How to Construct a Productive, Disciplined, Monoethnic Society. The Dilemma of East European Governments, 1914-1956 », in Amir Weiner, éd., Landscaping the Human Garden : Twentieth-Century Population Management in a Comparative Framework, Stanford : Stanford University Press, 2003, p. 205-217.

8. AJJDC, NY 192332 (Archives du Jewish Joint Distribution Committee, New York, section 1923-1932), 3/6/164, Activities of Refugee Department American Joint Distribution Committee in Europe during the Years 1921-1922-1923, p. 15.

9. Krasnonarmejcy v pol'skom plenu v 1919-1922 gg. Sbornik dokumentov i materialov [Les soldats de l'Armée rouge prisonniers des Polonais, 1919-1922. Recueil de documents], M. : Letnii sad, 2004, doc. 133, p. 282.

10. Ibid., doc. 210, p. 426.

11. Ibid., doc. 214, p. 428-429.

12. Leo Baeck Institute Archives, Emigration Collection, box 1, folder 4, «Mémoire de l'organisation sioniste de Pologne sur la question juive en Pologne» (19/01/1919) ; Alexander 
Victor Prusin, Nationalizing a Borderland: War, Ethnicity and Anti-Jewish Violence in East Galicia, 1914-1920, Tuscaloosa : University of Alabama Press, 2005.

13. Sur ces distinctions et la variété des traitements des déplacés, voir notamment: Gousseff, L'exil russe.

14. ASdN (Archives de la Société des Nations), fond Nansen, R1726, doc $n^{\circ} 15525$, "Mémoire relatif aux réfugiés juifs russes adressé au Haut Commissaire de la Société des Nations, pour les réfugiés russes par le comité exécutif de la Conférence universelle juive de secours ». À noter que les chiffres de victimes présentés à la SDN, après de nombreux tâtonnements, sont aujourd'hui ceux sur lesquels l'on s'accorde généralement, tant à la lumière des archives soviétiques que celles des organisations humanitaires.

15. AJJDC NY191921, 4/35/3/245.1, « Roumanian Letter n 407 »; AJJDC NY191921, 4/35/3/242.3, Télégramme de la Conférence juive universelle de secours (13/12/1920).

16. ASdN, fond Nansen, R1726, doc. $n^{\circ} 29025$, « Rapport au Dr Nansen » (23/05/1923).

17. ASdN, fond Nansen, R1715, doc. $n^{\circ} 32942 X$, «Organisation des secours alimentaires et de l'instruction des réfugiés en Pologne ».

18. AJJDC NY191921, 4/35/3/245.1, « Report on refugees - Bessarabian Section ».

19. Michael R. Marrus, The Unwanted : European Refugees from the First World War through the Cold War, Philadelphie: Temple University Press, 2002, p. 64 ; Pour la Roumanie, voir plus généralement les nombreuses coupures de journaux et déclarations rassemblées dans : YIVO Institute for Jewish Research (désormais YIVO), fond Lemuel Levitt, box 1.

20. ASdN, fond Nansen, R1725, doc. $n^{\circ} 25659$, «Le gouvernement et les éléments étrangers » (entretien avec le Président du Conseil des ministres, Sikorski, 14/02/1923).

21. Peter Gatrell, «War, Population Displacement and State Formation in the Russian Borderlands, 1914-24» in Nick Baron, Peter Gatrell, éds., Homelands : War, Population and Statehood in Eastern Europe and Russia 1918-1924, Londres : Anthem Press, 2004, p. 24.

22. ASdN, fond Nansen, R1725, doc. $n^{\circ} 25659$, « Threatened Expulsions of non Political Russian Refugees from Poland »(06/03/1923).

23. Sabine Dullin, «L'entre-voisins en période de transition étatique (1917-1924). La frontière épaisse des bolcheviks et à l'Est de l'Europe ", Annales HSS, 2 (2014), p. 383-414. (Merci à l'auteur de m'avoir permis d'y avoir accès avant sa sortie.)

24. Terry Martin, The Affirmative Action Empire : Nations and Nationalism in the Soviet Union, 1923-1939, Ithaca : Cornell University Press, 2001.

25. ASdN, fond Nansen, R1726, doc. n² 29025, Rapport du délégué en Pologne du HCR, 22/05/1923.

26. Krasnonarmejcy v pol'skom plenu, doc. 302, p. 602.

27. Ibid., doc. 338, p. 703-715.

28. Ibid., doc. 324, p. 675-676.

29. ASdN, fond Nansen, R1715, doc $n^{\circ} 32942 \mathrm{X}$, « Report of the representative in Russia of the High Commission for Refugees » (13/02/1924).

30. TsDAVO (Archives centrales d'État des organes de pouvoir et de gouvernement d'Ukraine), f. 4, NKID, op. 1, d. 556, ark. 84-85ob (4/1/84-85ob).

31. Le rapport du délégué polonais aux rapatriements est relayé par le HCR et d'autres organisations d'aide aux réfugiés. Ici cité de : AJJDC, NY 192332, 3/6/164, Activities of Refugee Department..., p. 19.

32. ASdN, fond Nansen, R1715, doc $n^{\circ} 32942 \mathrm{X}$, « Report of the representative in Russia of the High Commission for Refugees » (13/02/1924).

33. ASdN, fond Nansen, R1726, doc $n^{\circ} 16531$, «Données statistiques concernant les réfugiés juifs d'Ukraine se trouvant en Bessarabie "; Russo-Jewish Refugees in Eastern Europe.

34. TsDAVO 4/1/556/31ob, Tableau récapitulatif pour juillet 1921.

35. TsDAVO f. 5, NKVD, op. 1, d. 1190, ark. 13 (5/1/1190/13), Télégramme du bureau du NKVD de Volhynie (23/12/1921). 
36. Leo Baeck Institute Archives, Emigration Collection, box 1, folder 5, "Bulletin of the Executive Committee of the Jewish World Relief Conference ", $\mathrm{n}^{\circ}$ 1, 20/09/1921.

37. ASdN, fond Nansen, R1726, doc. $n^{\circ}$ 25659, « Russian refugees in Poland » (reçu le 30/04/1923). 38. ASdN, fond Nansen, R1725, doc. $n^{\circ} 25659$, Copie de la motion présentée à la Diète polonaise par des députés juifs sur la question des réfugiés, 27/02/1923.

39. AJJDC, NY192132, 3/6/164, "Activities of the Refugee Department. American Joint Distribution Committee in Europe during the years 192119221923 », p. 40, 61.

40. Dullin, « L'entre-voisins en période de transition étatique (1917-1924)... ».

41. AJJDC, NY192132, 3/6/164, « Activities of the Refugee Department... », p. 40.

42. ASdN, fond Nansen, R1715, doc. $n^{\circ} 32942 X$, « Rapport sur l'activité du Haut Commissariat pour les réfugiés en Pologne depuis le $1^{\text {er }}$ juillet 1923 » et son annexe « Répartition approximative des cartes d'asile et de séjour ».

43. AJJDC, NY192132, 3/6/164, "Activities of the Refugee Department...", p. 40-42; Leo Baeck Institute Archives, Emigration Collection, box 1, folder 5, «Bulletin of the Executive Committee of the Jewish World Relief Conference ", $n^{\circ} 2,17 / 11 / 1921$.

44. Leo Baeck Institute Archives, Emigration Collection, box 1, folder 5, "Bulletin of the Executive Committee of the Jewish World Relief Conference », $\mathrm{n}^{\circ} 3,06 / 02 / 1922$.

45. Ibid., p. 42-43.

46. Konrad Zielinski, «Population Displacement and Citizenship in Poland, 1918-24 » in Baron, Gatrell, éds., Homelands, p. 107.

47. ASdN, fond Nansen, R1714, doc. $\mathrm{n}^{\circ}$ 19252, «Réfugiés Russes. Rapport général sur l'œuvre accomplie jusqu'au 15 mars 1922 par M. Fridtjof Nansen, Haut Commissaire de la Société des Nations "; ASdN, fond Nansen, R1725, doc. $n^{\circ} 21783$, "Mémoire sur les réfugiés russes en Pologne » (07/07/1922).

48. ASdN, fond Nansen, R1725, doc $n^{\circ} 25659$, «Memorandum» (23/03/1923) ; et plus généralement : AJJDC, NY192132, 3/6/164, « Activities of the Refugee Department... », p. 43-44.

49. ASdN, fond Nansen, R1715, doc. $n^{\circ} 29424$, «Réfugiés russes. Rapport du Dr Nansen » (reçu le 26/07/1923).

50. ASdN, fond Nansen, R1726, doc. $n^{\circ} 25659$, Lettre du délégué de la Conférence universelle juive de secours auprès des institutions internationales à Genève, 23/04/1923.

51. Voir par exemple le télégramme du général Sikorski au Dr Nansen du 14 avril 1923 vantant l'accueil des réfugiés par "humanitarisme traditionnel Polonais» (sic) et la souplesse de la politique du gouvernement face à des réfugiés qui profitent de lui : ASdN, fond Nansen, R1726, doc. $n^{\circ} 25659$; et la lettre du responsable du HCR: ASdN, fond Nansen, R1726, doc. $n^{\circ} 29025$, Rapport du délégué en Pologne du HCR, 22/05/1923.

52. ASdN, fond Nansen, R1725, doc. $\mathrm{n}^{\circ} 25659$, «Le gouvernement et les éléments étrangers » (entretien avec le Président du Conseil des ministres, Sikorski, 14/02/1923).

53. ASdN, fond Nansen, R1725, doc. $n^{\circ} 25659$, Télégramme de Varsovie au HCR, 19/02/1923.

54. AJJDC, NY192132, 3/6/164, "Activities of the Refugee Department... ", p. 44; ASdN, fond Nansen, R1726, doc. $n^{\circ}$ 25659, Lettre au délégué en Pologne du HCR, 05/10/1923.

55. ASdN, fond Nansen, R1726, doc. $n^{\circ} 25659$, Annexe à la lettre du 27 août 1923 sur la situation de l'émigration des réfugiés juifs.

56. ASdN, fond Nansen, R1715, doc. $n^{\circ} 31937$, Notes générales sur les réfugiés par le HCR (26/10/1923).

57. ASdN, fond Nansen, R1725, doc. $n^{\circ} 25659$, Copie de la motion présentée à la Diète polonaise par des députés juifs sur la question des réfugiés, 27/02/1923.

58. ASdN, fond Nansen, R1714, doc. $n^{\circ} 20855$, Décret d'amnistie du 13/11/1921 ; Gousseff, L'exil russe, p. 86-87.

59. ASdN, fond Nansen, R1726, doc. $n^{\circ} 27915$, « Note sur la situation des réfugiés russo-juifs en Europe orientale... » 
60. ASdN, fond Nansen, R1726, doc. $n^{\circ} 25659$, Lettre du délégué en Pologne du HCR, 14/04/1923.

61. ASdN, fond Nansen, R1726, doc $n^{\circ} 25659$, Annexe à la lettre du 27 août 1923.

62. TsDAVO 5/1/1190/13.

63. Sabine Dullin, «Les protecteurs. Le rôle des gardes-frontières dans la surveillance des frontières occidentales de l'URSS (1917-1939) », in Sophie Cœuré, Sabine Dullin, éds., Frontières du communisme : Mythologies et réalités de la division de l'Europe de la révolution d'Octobre au mur de Berlin, P. : La Découverte, 2007, p. 379-405.

64. AJJDC, NY192132, 4/30/3/487, « Refugee situation and refugee relief in Russia ».

65. Ibid.

66. Le partenariat avec l'ARA est documenté dans: H.H. Fisher, The Famine in Soviet Russia 1919-1923 : The Operation of the Americian Relief Association, New York : Macmillan, 1927. Pour un rapide panorama de cette action du JDC en Europe orientale, voir : Moses A. Leavitt, L'histoire de l'A.J.D.C. : 1914-1952, New York : Americain Joint Distribution Committee, 1953.

67. AJJDC, NY192132, 4/30/3/487, « Refugee situation... ».

68. AJJDC, NY192132, 4/30/1/458, "Report of the activities of the Joint Distribution Committee (Relief Department). Russia» (du 01/11/1923 au 31/10/1923), p. 59-60 ; Krasnonarmejcy v pol 'skom plenu, doc. 285, p. 559.

69. AJJDC, NY192132, 4/30/3/487, « Refugee situation... ».

70. TsDAVO 4/1/32/25-25ob, Lettre du délégué du NKID SSSR à Čubar, président du Conseil des commissaires du peuple de RSSU (début novembre 1923).

71. RGASPI (Archives d'État de Russie de l'histoire sociale et politique), f. 19, op. 3, d.208, 1. 179-180. (19/3/208/179-180), Rapport de Dzeržinskij au STO RSFSR.

72. Krasnonarmejcy v pol'skom plenu, doc. 586, p. 560-561.

73. Ibid., doc. 556, p. 285.

74. TsDAVO 5/5/100, passim.

75. AJJDC, NY192132, 4/30/1/458, « Report... », p. 59-60.

76. Kateryna Stadnik, «The Repatriation of Polish Citizens from Soviet Ukraine to Poland in 1921-2 » in Baron, Gatrell, éds., Homelands, p. 122-123.

77. AJJDC, NY192132, 4/30/1/458, « Report... », p. 60.

78. AJJDC, NY192132, 4/30/3/487, "Refugee situation...»; les talons des laissez-passer sont adjoints aux brèves enquêtes individuelles : TsDAVO 5/5/100, passim.

79. Nicolas Werth, "Le "nettoyage" des frontières soviétiques dans les années 1930 ", in Cœuré, Dullin, éds., Frontières du communisme, p. 358-378.

80. Radu Ioanid, La Roumanie et la Shoah : Destruction et survie des Juifs et des Tsiganes sous le régime Antonescu (1940-1944), P. : Éditions de la Maison des Sciences de l'homme, 2002.

81. Sur toutes ces questions de rapatriements vers l'Union soviétique, voir Catherine Gousseff, «Des migrations de sortie de guerre qui reconfigurent la frontière : ouverture et fermeture de l'URSS avant la guerre froide ", in Cœuré, Dullin, éds., Frontières du communisme, p. 428-442.

\section{RÉSUMÉS}

L'article se concentre sur les déplacements de populations juives d'Ukraine, organisés, spontanés et forcés, qui ont suivi la fin de la guerre civile et de la guerre soviéto-polonaise en 1920. Il montre comment les nouveaux États, polonais et roumain, ont fait de la présence de minorités 
juives originaires de la future Union soviétique un problème public, une menace politique et socio-économique, devant être réglé par des pratiques de contrôle et de retours forcés puis de déportation. Le phénomène s'inscrit ainsi dans le cadre de l'affirmation des nouvelles frontières nées de la guerre en Europe orientale, et d'une gestion des minorités juives marquée par un antisémitisme aggravé et les rêves d'États-nations homogènes et forts. Enfin, l'article analyse l'accueil des Juifs de retour en Ukraine soviétique et les voies singulières des politiques humanitaires soviétiques, abandonnant aux organisations juives le soin des réfugiés et orchestrant de vastes opérations de contrôle sur des populations jugées suspectes.

The article focuses on spontaneous and state-organized mass displacements of Ukrainian Jewish populations after the civil war and the 1920 Soviet-Polish war. It shows how the new Polish and Rumanian states turned the presence of Jewish minorities from the future Soviet Union into a public problem, a political and socio-economic threat that had to be settled by control, forcible returns, and ultimately, deportation. This phenomenon took place against the backdrop of the affirmation of new post-war borders in Eastern Europe and the treatment of Jewish minorities marked by strong anti-Semitism in would-be strong and homogeneous nation-states. Last, the article analyzes how returning Jews were greeted in Soviet Ukraine and describes the singular Soviet humanitarian practices consisting in handing over the care of refugees to Jewish organizations and orchestrating extensive control operations on suspect populations.

\section{AUTEUR}

\section{THOMAS CHOPARD}

CERCEC - EHESS, chopard@ehess.fr 